\title{
Thermophysical and Magnetic Properties of Carbon Beads Containing Nickel Nanocrystallites
}

\author{
A. Skumiel - M. Izydorzak • M. Leonowicz • \\ A. D. Pomogailo - G. I. Dzhardimalieva
}

Received: 22 October 2010 / Accepted: 21 June 2011 / Published online: 7 July 2011

(C) The Author(s) 2011. This article is published with open access at Springerlink.com

\begin{abstract}
Ferromagnetic and superparamagnetic nickel nanocrystallites, stabilized in a carbon matrix, were prepared by a three-step procedure including formation of a Ni acrylamide complex, followed by frontal polymerization and pyrolysis of the polymer at various temperatures. It was found that the procedure applied enables fabrication of magnetic beads containing metallic nanocrystallites embedded in a carbon matrix. The size of the crystallites, their morphology, volume fraction, and magnetic properties can be tailored by the pyrolysis temperature. The size of the crystallites affects their behavior in an external magnetic field, i.e., a heating process is the most effective for a sample pyrolyzed at $873 \mathrm{~K}$. The revealed $H^{\mathrm{n}}$-type dependence of the temperature increase rate, $(\mathrm{d} T / \mathrm{d} t)_{t=0}$, on the amplitude of the magnetic field indicates the presence of both superparamagnetic and ferromagnetic particles in all the samples studied since $n>2$. For the superparamagnetic particles, the heating mechanism is associated with Néel relaxation. For the lower values of the magnetic field amplitude, $H<H_{0}$, the relaxation losses dominate whereas for the opposite case, $H>H_{0}$, the magnetic hysteresis is the main source of thermal energy losses. The composites containing magnetic Ni nanocrystallites entrapped in a carbon matrix can be potentially applied for hyperthermia treatment.
\end{abstract}

\footnotetext{
A. Skumiel

Institute of Acoustics, Faculty of Physics, Adam Mickiewicz University,

Umultowska 85, 61-614 Poznan, Poland

e-mail: skumiel@amu.edu.pl

M. Izydorzak $(\varangle) \cdot$ M. Leonowicz

Faculty of Materials Science and Engineering, Warsaw University of Technology,

Woloska 141, 02-507 Warsaw, Poland

e-mail: mizydorz@inmat.pw.edu.pl
}

A. D. Pomogailo · G. I. Dzhardimalieva

Institute of Chemical Physics RAS, Chernogolovka, Russia 
Keywords Heating process $\cdot$ Magnetic beads $\cdot$ Néel relaxation

\section{Introduction}

When a sample, which contains monodomain magnetic nanoparticles, is placed into an external alternating magnetic field, the magnetic vector $M$ in that sample undergoes reorientation in space following the magnetic field intensity vector $H$. As a result of the phase delay of the magnetic vector $M$, in relation to the vector $H$, a part of the magnetic field energy undergoes irreversible conversion into thermal energy. It leads to heating of the magnetic sample, which can be used in biomedicine for therapy that destroys cancerous cells.

This so-called hyperthermia relies on the heating of specific body organs or tissues to temperatures of $42{ }^{\circ} \mathrm{C}$ to $45^{\circ} \mathrm{C}$ for about $20 \mathrm{~min}$ to $30 \mathrm{~min}$.

In practice, a few reasons exist for application of an alternating magnetic field energy loss and its exchange for thermal energy. If the sample contains only superparamagnetic particles, magnetic relaxation appears and such a sample magnetizes itself according to two mechanisms: by Brown and by Néel relaxation.

The Brown mechanism involves rotations of the entire magnetic particle relative to the surrounding medium while the Néel mechanism is due to reorientation of the magnetization vector within the particle. The thermal losses corresponding to Néel and/or Brownian relaxation are proportional to the square of the magnetic field strength.

The Brown mechanism can only occur when magnetic particles have freedom of rotation such as the case of a magnetic fluid. Then the value of the relaxation time is dependent on the absolute viscosity of the fluid, the hydrodynamic volume of magnetic particles, and the temperature. When these magnetic particles are in gels or in a solid medium and there is no possibility to arrange towards an external magnetic field, then the relaxation time $\tau_{\mathrm{B}} \rightarrow \infty$. But even then such sample undergoes a magnetization thanks to the Néel mechanism which is due to reorientation of the magnetization vector within the particle.

Each of these mechanisms is defined by the appropriate average relaxation time according to the well-known expressions [1]:

$$
\tau_{\mathrm{B}}=\frac{3 \eta_{\mathrm{S}} V_{\mathrm{h}}}{k_{\mathrm{B}} T} \quad \text { and } \quad \tau_{\mathrm{N}}=\tau_{0} \exp \left(\frac{K V}{k_{\mathrm{B}} T}\right)
$$

where $V_{\mathrm{h}}$ is the hydrodynamic volume of the magnetic grain, $k_{\mathrm{B}}=1.38 \times 10^{-23} \mathrm{~J} \cdot \mathrm{K}^{-1}$ is the Boltzmann constant, $T$ is the absolute temperature, $\eta_{\mathrm{S}}$ is the shear viscosity coefficient of carrier fluid, $\tau_{0}$ is the time constant $1 \mathrm{~ns}, K\left(\mathrm{~J} \cdot \mathrm{m}^{-3}\right)$ is the anisotropy constant for the magnetic material, and $V$ is the volume of the magnetic grain.

A characteristic feature of thermal energy losses in AC magnetic field results from a relaxation process and then the volumetric power dissipation is proportional to the square of the magnetic field strength, $H[2]$ :

$$
P(f, H)=\pi \mu_{0} \chi_{0} f H^{2} \frac{2 \pi f \tau}{1+(2 \pi f \tau)^{2}},
$$


where $\chi_{0}$ is the equilibrium susceptibility of a magnetic sample, $f$ is the frequency of the alternate magnetic field, $\tau$ is the relaxation time, and $\mu_{0}=4 \pi \times 10^{-7} \mathrm{~V} \cdot \mathrm{s}$. $\mathrm{A}^{-1} \cdot \mathrm{m}^{-1}$ is the permeability of free space.

The imaginary part of the magnetic susceptibility is a function of both the frequency and magnetic field strength $H_{0}$ according to the following equation [2]:

$$
\chi^{\prime \prime}=\chi_{\mathrm{o}} \frac{\omega \tau}{1+(\omega \tau)^{2}}=\frac{\mu_{\mathrm{o}} \phi M_{\mathrm{S}}^{2} V}{k_{\mathrm{B}} T} \frac{L(\xi)}{\xi} \frac{\omega \tau}{1+(\omega \tau)^{2}},
$$

where $\chi_{\mathrm{o}}=3 \chi_{i}\left(\operatorname{coth} \xi-\xi^{-1}\right) \xi^{-1}$ is the equilibrium value of the magnetic fluid susceptibility, $\chi_{i}=(\partial M / \partial H)_{i}$ is the initial susceptibility determined from differentiation of the Langevin relationship, $\phi$ is the volume fraction of particles in the suspension, $M_{\mathrm{S}}$ is the saturation magnetization of the magnetic material, and $\xi=\mu_{\mathrm{o}} M_{\mathrm{S}} V H(k T)$ is the parameter of the Langevin function $L(\xi)$. From Eq. 3 it follows that for $\omega \tau=1$, the imaginary part of the magnetic susceptibility $\chi^{\prime \prime}$, achieves the maximal value.

Because of polydispersion, magnetic nanoparticles with small sizes are located in the sample, being the source of relaxation losses, but there are also particles of larger sizes, which are the source of energy losses for hysteresis. From Refs. [3-6] it follows that for particle systems with ferromagnetic behavior (i.e., hysteresis), a power of three is found at low field amplitudes (for Rayleigh losses). Thus, hysteresis losses, for so-called Rayleigh loops, may be well described by a third-order power law. In that case, it can be written as $P \propto H^{3}$.

In this investigation magnetic beads, containing nickel nanocrystallites, were prepared and studied in the light of their potential applications in magnetic heating.

\section{Experimental}

The procedure of preparation of Ni containing nanocomposites consisted of three steps. In the first step, the acrylamide metal complex was obtained by a substitution reaction of inorganic hydrates by the acrylamide. Afterwards, frontal polymerization was carried out at atmospheric pressure in a self-generated atmosphere. The third step, i.e., pyrolysis of the polymeric matrix, enabled obtaining of nanocomposite powder particles. The size of the Ni nanoparticles, entrapped in a carbon matrix, depended on the condition of the pyrolysis, mostly on the temperature of the process. A more detailed description of the processing route can be found in [7].

X-ray diffraction (XRD) patterns were obtained using a Rigaku MiniFlexII X-ray diffractometer equipped with a copper cathode $\left(\lambda\left(\mathrm{Cu} \mathrm{K}_{\alpha}\right)=0.154184 \mathrm{~nm}\right)$, in a scan range $(2 \theta)$ from $20^{\circ}$ to $100^{\circ}$. Additionally, Raman spectra were studied using a Raman spectrometer (Nicolet Almega XR, laser excitation wavelength of $532 \mathrm{~nm}$ ).

The microstructure was observed using a scanning electron microscope (SEM, Hitachi S-3500N) and analyzed using a scanning transmission electron microscope (STEM, JEOL JEM 1200 EX).

The hysteresis loops were recorded at room temperature, in an external magnetic field of $\pm 1600 \mathrm{kA} \cdot \mathrm{m}^{-1}$. Zero-field-cooled (ZFC) and field-cooled (FC) magnetization curves were recorded as a function of temperature in a range of $5 \mathrm{~K}$ to $300 \mathrm{~K}$, in 
a field of $8 \mathrm{kA} \cdot \mathrm{m}^{-1}$. In the FC regime, the specimens were cooled to $5 \mathrm{~K}$ in a field of $8 \mathrm{kA} \cdot \mathrm{m}^{-1}$ and subsequently heated in the same field to room temperature. In the $\mathrm{ZFC}$ regime, cooling was performed without the magnetic field.

The magnetic field frequency $f=450 \mathrm{kHz}$ was selected for the sake of the maximum conversion efficiency of the hyperthermal phenomenon. The temperature of the sample was monitored using a thermocouple (LT Lutron TM-917) [8] with an absolute accuracy of $\pm 0.01^{\circ} \mathrm{C}$.

The heating system consisted of a broadband power amplifier, an induction coil (length of $78 \mathrm{~mm}$ ), and an oscilloscope (Philips). An induction coil formed part of an $R L C$ series circuit (self-inductance $L_{\mathrm{o}}=15.6 \mu \mathrm{H}$ in air; without a magnetic sample). The magnetic field strength was determined on the basis of a voltage measurement for a dropping resistor $(R=1 \Omega)$ at voltage resonance (oscilloscope reading).

A glass tube containing the sample was thermally isolated by a layer of fabric from the solenoid winding supported on a plastic sleeve. Water $(0.1 \mathrm{~mL})$ was added to the vial with magnetic material to provide for better thermal contact with the thermocouple.

\section{Results and Discussion}

\subsection{Structure and Magnetic Properties}

In this section, we discuss properties of the specimens after the complete, three-step processing route, because only these materials exhibit magnetic properties. Both the monomer and polymer obtained after the first and second step, respectively, are nonmagnetic.

The beads after pyrolysis, independent of the applied temperature, were in a form of irregular particles, having sizes ranging from a few $\mu \mathrm{m}$ and up to $300 \mu \mathrm{m}$ (Fig. 1).

Application of Raman spectroscopy for the beads revealed that the matrix of the particles was made up of both amorphous carbon and graphite ( $\mathrm{G}$ and $\mathrm{D}$ lines characteristic of these forms of carbon) (Fig. 2). Additionally the D/G ratio gives information on the crystallinity of the sample; thus, the lower the D/G ratio, the better the ordering of the graphite structure [9]. For our specimen pyrolyzed at $873 \mathrm{~K}$, a D/G ratio of
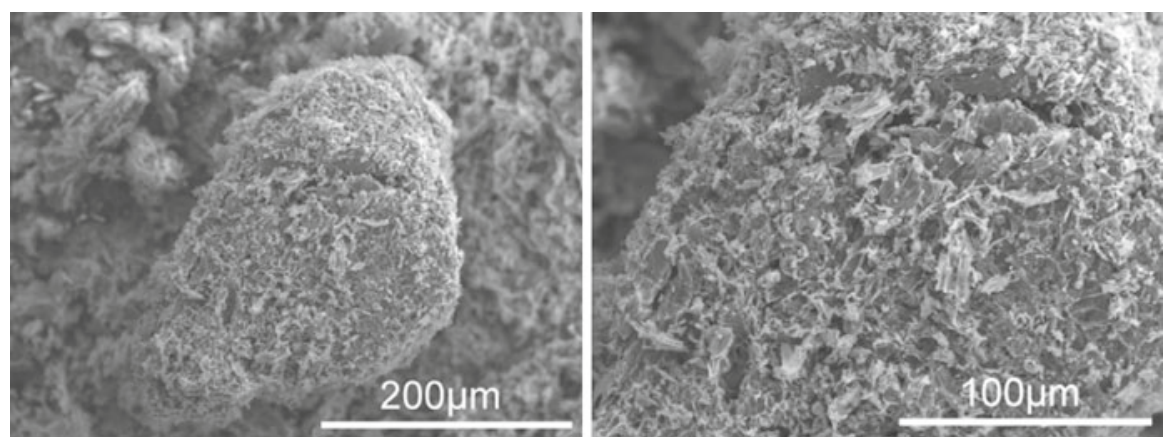

Fig. 1 SEM microstructure of the pyrolyzed material, pyrolysis temperature of $773 \mathrm{~K}$ 


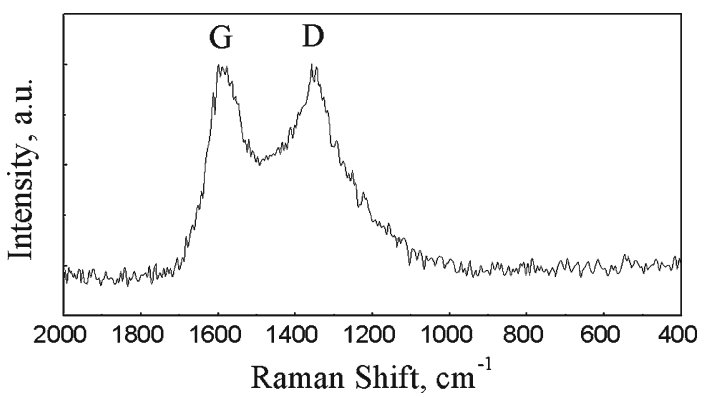

Fig. 2 Raman spectrum of the material pyrolyzed at $873 \mathrm{~K}$

about 1 was revealed, indicating the presence of an imperfect graphite structure with a high amount of structural defects.

X-ray phase analysis revealed that the metallic Ni (FCC) was the main phase identified for all the pyrolysis temperatures (Fig. 3). The part of a diffused halo for samples pyrolyzed at $673 \mathrm{~K}$ and $773 \mathrm{~K}$, at $2 \theta$ close to $20^{\circ}$, represents amorphous carbon [10]. The change in the shape of this peak, observed for the NiAAm873 sample, can indicate that application of a higher pyrolysis temperature leads to formation of the crystalline form of carbon (graphitized carbon) in the matrix structure [11]. The diffraction peaks, originated from the nanocrystallites in the beads, were broadened, which points to their nanocrystalline structure. The higher the pyrolysis temperature, the narrower and more intensive were the reflections, what can be attributed to the higher amount of the magnetic component.

An observation using transmission electron microscopy (TEM) provided information concerning morphology of the Ni nanocrystallites (Fig. 3). The crystallite sizes, measured form the micrographs, were $6 \mathrm{~nm}$ to $7 \mathrm{~nm}$ and $8 \mathrm{~nm}$ to $14 \mathrm{~nm}$, for $673 \mathrm{~K}$ and $773 \mathrm{~K}$, respectively. For $873 \mathrm{~K}$ the crystallites grew slightly and the size range was somewhat broader, $10 \mathrm{~nm}$ to $17 \mathrm{~nm}$.

The size and volume fraction of the nanocrystallites determine the magnetic properties of the beads. On the basis of hysteresis loops for materials pyrolyzed at $673 \mathrm{~K}, 773$ $\mathrm{K}$, and $873 \mathrm{~K}$, respectively, recorded at room temperature (Table 1), the basic magnetic properties such as the saturation magnetization $\left(M_{\mathrm{S}}\right)$, coercivity $\left(\mathrm{J} H_{\mathrm{C}}\right)$, and remanence $\left(M_{\mathrm{R}}\right)$ were assessed. The value of the saturation magnetization enabled assessing the mass fractions of the metallic components $\left(F_{\mathrm{mc}}\right)$, being $(7,21$, and 32$)$ mass $\%$, for pyrolysis temperatures $673 \mathrm{~K}, 773 \mathrm{~K}$, and $873 \mathrm{~K}$, respectively. The specimens pyrolyzed at two lower temperatures showed ${ }_{\mathrm{J}} H_{\mathrm{C}}$ and $M_{\mathrm{R}}$ close to zero, indicating the lack of magnetic anisotropy and domination of superparamagnetic ordering, whereas the material pyrolyzed at $873 \mathrm{~K}$ shows ferromagnetic interactions. Moreover, a quite high coercivity may indicate some dissolution of carbon in the nickel lattice, as was previously found for Ni nanocrystallites processed by arc discharge [12].

In order to determine the blocking temperature $T_{\mathrm{B}}$, the ZFC and FC magnetizations as a function of temperature were measured for all three specimens, in an external field $8 \mathrm{kA} \cdot \mathrm{m}^{-1}$, within the temperature range of $5 \mathrm{~K}$ to $300 \mathrm{~K}$ (Fig. 4). Above $T_{\mathrm{B}}$, the material experiences high thermal fluctuations and exhibits superparamagnetic properties. 


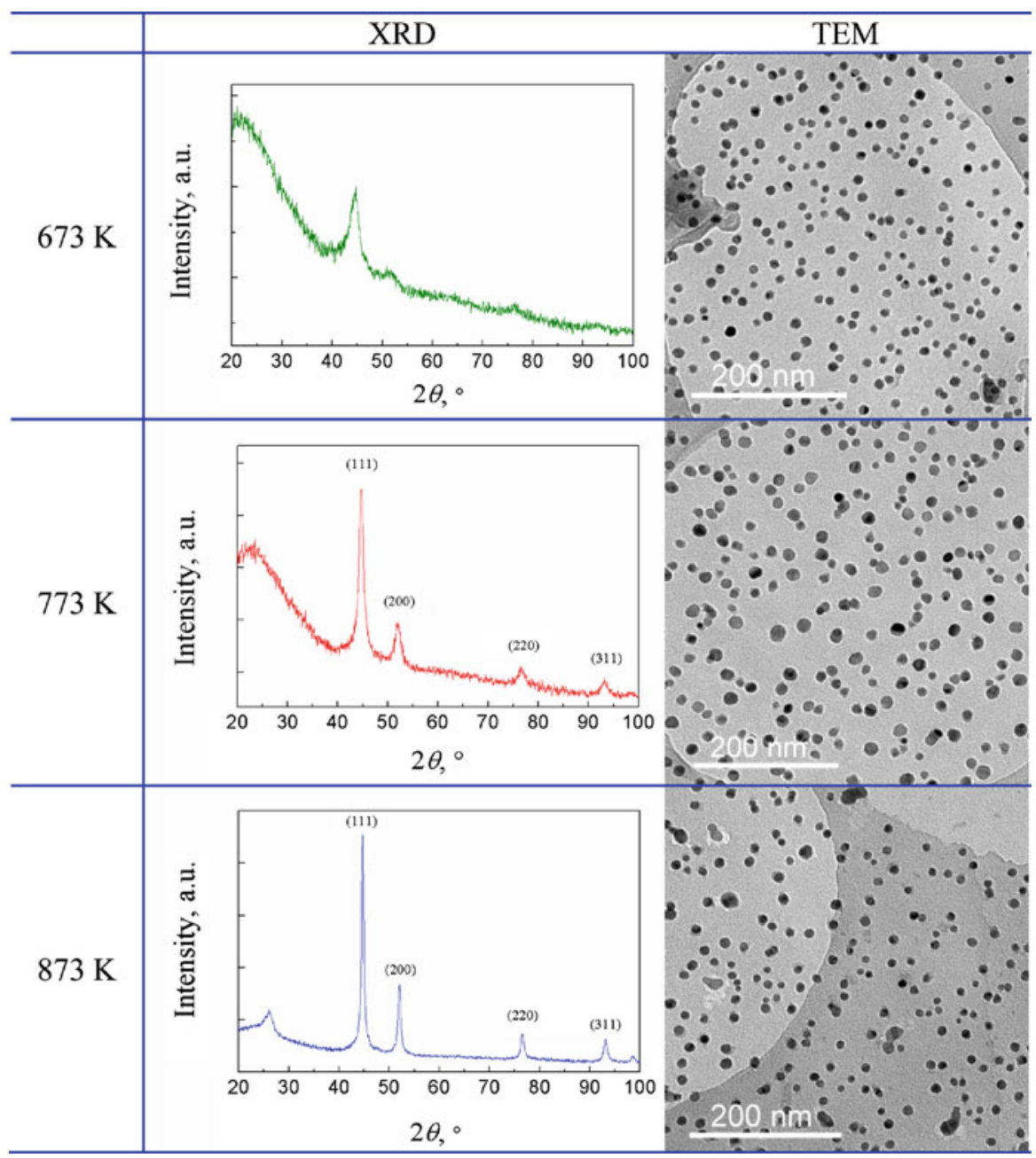

Fig. 3 XRD patterns and TEM images of Ni-beads obtained at different pyrolysis temperatures

Below $T_{\mathrm{B}}$, the effect of thermal energy decreases and ferromagnetic ordering returns. The results of measurement of the FC and ZFC curves, for the specimen pyrolyzed at $673 \mathrm{~K}$ and $773 \mathrm{~K}$, show a behavior characteristic of a superparamagnetic material. The maximum in the ZFC magnetization indicates the blocking temperature $T_{\mathrm{B}}$ (temperature at which the time for thermal activation of the magnetic moments over the energy barrier becomes comparable to the measurement time) and amounts to $45 \mathrm{~K}$ and $120 \mathrm{~K}$, for the specimen obtained at $673 \mathrm{~K}$ and $773 \mathrm{~K}$, respectively. However, in the latter case the ZFC peak is very broad, indicating that the nanocrystallites have a wide distribution of sizes and blocking temperatures. As was noted, the maximum of the ZFC magnetization defines the mean blocking temperature. One has to remember that $T_{\mathrm{B}}$ depends on several parameters such as the magnetic field strength, material type, 
Table 1 Room temperature hysteresis loops and basic magnetic properties of Ni-beads pyrolyzed at $673 \mathrm{~K}, 773 \mathrm{~K}$, and $873 \mathrm{~K}$

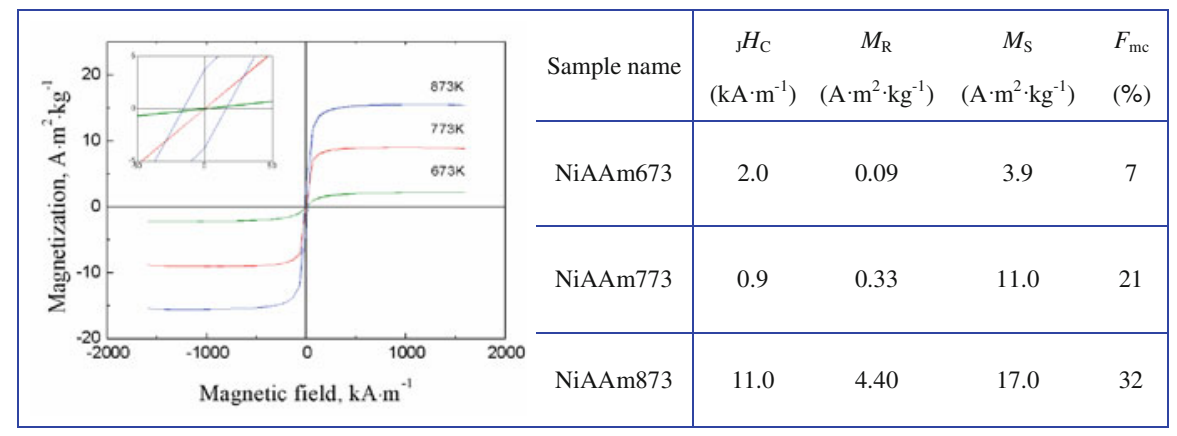
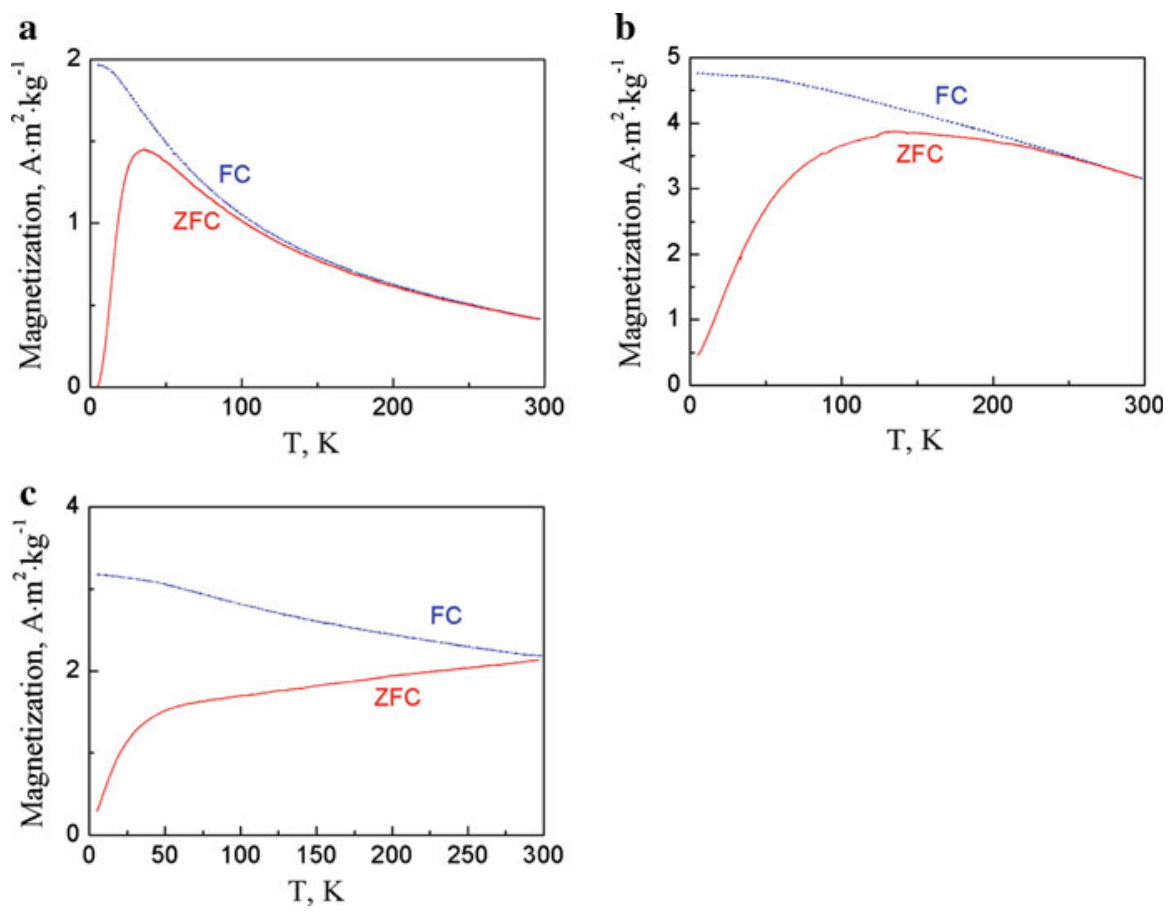

Fig. 4 FC and ZFC curves for specimens pyrolyzed at (a) $673 \mathrm{~K}$, (b) $773 \mathrm{~K}$, and (c) $873 \mathrm{~K}$ for magnetic field of $8 \mathrm{kA} \cdot \mathrm{m}^{-1}$

and crystallite size [13]. Above the temperature at which both curves, ZFC and FC, coincide, a state of thermodynamic equilibrium for magnetization relaxation occurs and the material becomes superparamagnetic [14-16].

The material pyrolyzed at $873 \mathrm{~K}$ presents a different behavior. The shape of both curves indicates that the material properties are controlled by magnetostatic interactions and the nanocrystallites show ferromagnetic ordering. 
For our composites containing Ni nanocrystallites, the crystallite size of about 10 $\mathrm{nm}$ seems to be the threshold where the ferromagnetism-superparamagnetism transition occurs. However, the diffusion of carbon to the nickel lattice and agglomeration of nanocrystallites are not irrelevant.

\subsection{Calorimetric Experiments}

Figure 5 presents the time changes of the magnetic sample temperature, for samples subjected to an alternate magnetic field of different strength $H_{\mathrm{AC}}$ and a frequency of $f=450 \mathrm{kHz}$.

The course of the curve $T(t)$ is a measure of the power released in a unit volume of the sample.

Figure 6 presents the rate of temperature increase $(\mathrm{d} T / \mathrm{d} t)_{t=0}$ at the beginning of the heating process as a function of the magnetic field intensity $H$ in the form of an exponential function:

$$
\left(\frac{\mathrm{d} T}{\mathrm{~d} t}\right)_{t=0}=\left(\frac{H}{a}\right)^{n}
$$
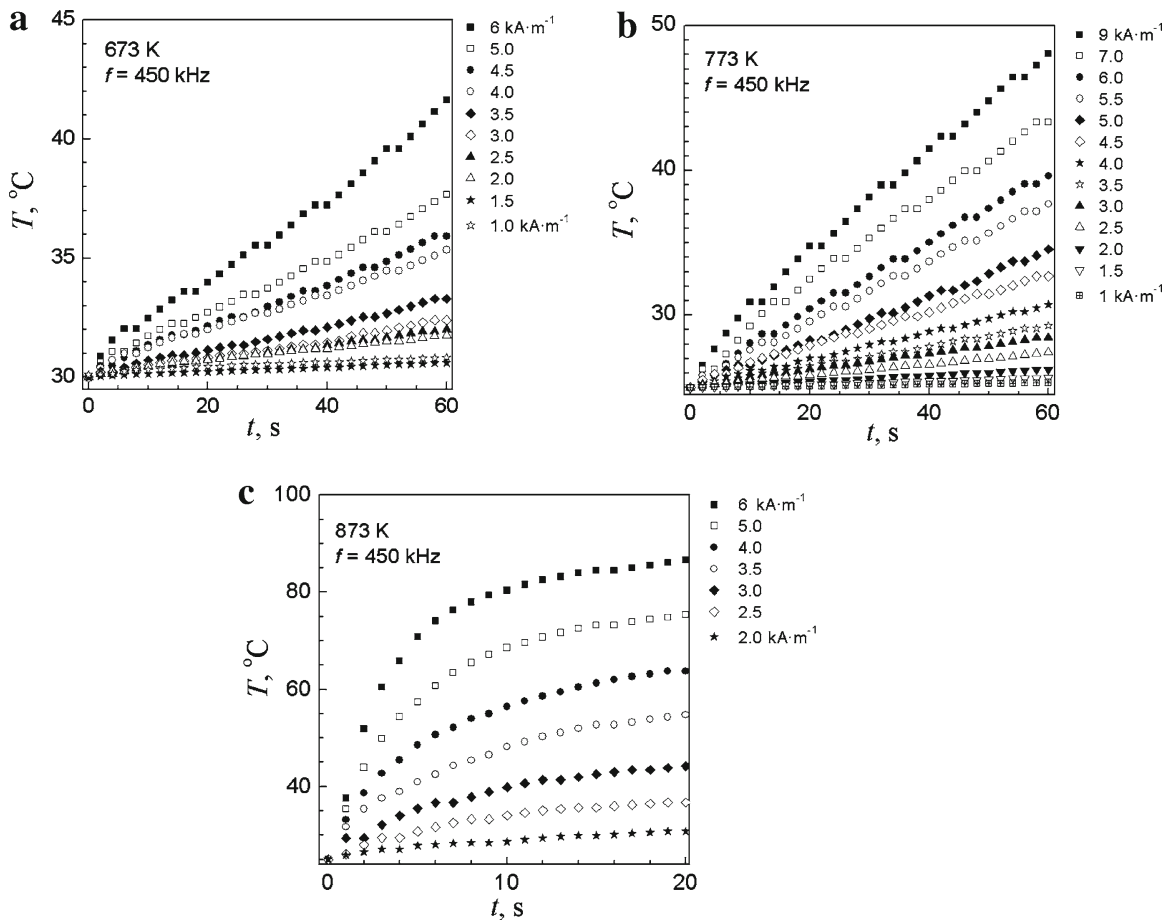

Fig. 5 Time-temperature curves for samples obtained at various pyrolysis temperatures: (a) $673 \mathrm{~K}$, (b) $773 \mathrm{~K}$, and (c) $873 \mathrm{~K}$, for different values of the magnetic field $H_{\mathrm{AC}}$, of frequency $f=450 \mathrm{kHz}$ 

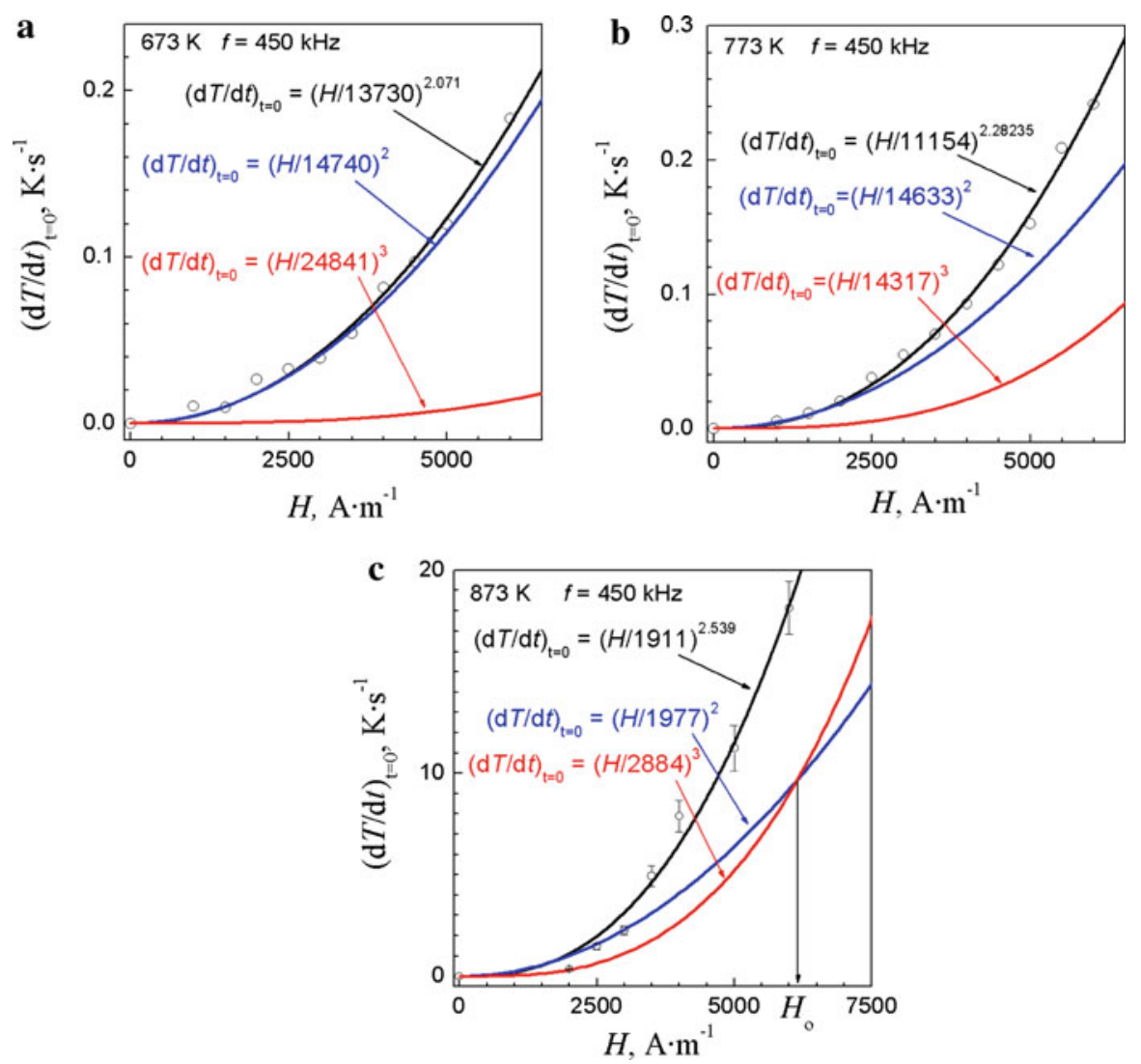

Fig. 6 Dependence of $(\mathrm{d} T / \mathrm{d} t)_{t=0}$ on the alternating magnetic field strength $H_{\mathrm{AC}}$ at a frequency $f=$ $450 \mathrm{kHz}$, for samples produced at (a) $673 \mathrm{~K}$, (b) $773 \mathrm{~K}$, and (c) $873 \mathrm{~K}$. Their constituents are derived from relaxation and hysteresis phenomena

where $a$ and $n$ are the parameters obtained from the fit of the exponential function, Eq. 4, to the experimental data.

Experimental values of the initial slope of the temperature increase rate $(\mathrm{d} T / \mathrm{d} t)_{t=0}$ are presented in Table 2.

The analysis of calorimetric results allowed an estimate of the contribution of relaxational and hysteresis loss mechanisms to total energy losses in the hyperthermal effect under the influence of an alternating magnetic field. Based on the principle of energy additivity, we can then write that the released power of losses is proportional to $(\mathrm{d} T / \mathrm{d} t)_{t=0}$ and consists of two components [17]:

$$
\left(\frac{\mathrm{d} T}{\mathrm{~d} t}\right)_{t=0}=\left(\frac{H}{a}\right)^{n}=\left(\frac{H}{r}\right)^{2}+\left(\frac{H}{h}\right)^{3}
$$

where $r$ and $h$ are parameters from the fit, which are adequate to describe losses for the relaxational mechanism and hysteresis. 
Table 2 Experimental values of the initial slope of the temperature increase rate $(\mathrm{d} T / \mathrm{d} t)_{t=0}\left(K \cdot \mathrm{s}^{-1}\right)$ for different values of the magnetic field strength

\begin{tabular}{|c|c|c|c|c|c|}
\hline \multicolumn{6}{|c|}{$(\mathrm{d} T / \mathrm{d} t)_{t=0} \quad f=450 \mathrm{kHz}$} \\
\hline \multicolumn{2}{|l|}{$673 \mathrm{~K}$} & \multicolumn{2}{|l|}{$773 \mathrm{~K}$} & \multicolumn{2}{|l|}{$873 \mathrm{~K}$} \\
\hline$H\left(\mathrm{~A} \cdot \mathrm{m}^{-1}\right)$ & $\overline{\left(K \cdot \mathrm{s}^{-1}\right)}$ & $H\left(\mathrm{~A} \cdot \mathrm{m}^{-1}\right)$ & $\left(K \cdot \mathrm{s}^{-1}\right)$ & $H\left(\mathrm{~A} \cdot \mathrm{m}^{-1}\right)$ & $\left(K \cdot \mathrm{s}^{-1}\right)$ \\
\hline 1000 & 0.0103 & 1000 & 0.0054 & 2000 & 0.36 \\
\hline 1500 & 0.0096 & 1500 & 0.0113 & 2500 & 1.474 \\
\hline 2000 & 0.0266 & 2000 & 0.0204 & 3000 & 2.258 \\
\hline 2500 & 0.0326 & 2500 & 0.038 & 3500 & 4.935 \\
\hline 3000 & 0.0393 & 3000 & 0.0549 & 4000 & 7.884 \\
\hline 3500 & 0.0543 & 3500 & 0.0704 & 5000 & 11.226 \\
\hline 4000 & 0.0815 & 4000 & 0.0928 & 6000 & 18.14 \\
\hline 4500 & 0.0970 & 4500 & 0.1220 & & \\
\hline 5000 & 0.1196 & 5000 & 0.1525 & & \\
\hline \multirow[t]{2}{*}{6000} & 0.1832 & 5500 & 0.2091 & & \\
\hline & & 6000 & 0.2416 & & \\
\hline
\end{tabular}

Table 3 Values of the parameters obtained from fitting Eq. 5 to the experimental data

\begin{tabular}{|c|c|c|c|c|c|}
\hline Sample name & $\begin{array}{l}a\left(\mathrm{~A} \cdot \mathrm{m}^{-1}\right. \\
\left.\mathrm{s}^{1 / n} \cdot \mathrm{K}^{-1 / n}\right)\end{array}$ & $n(-)$ & $\begin{array}{l}r\left(\mathrm{~A} \cdot \mathrm{m}^{-1}\right. \\
\left.\mathrm{s}^{0.5} \cdot \mathrm{K}^{-0.5}\right)\end{array}$ & $\begin{array}{l}h\left(\mathrm{~A} \cdot \mathrm{m}^{-1} .\right. \\
\left.\mathrm{s}^{1 / 3} \cdot \mathrm{K}^{-1 / 3}\right)\end{array}$ & $\begin{array}{l}H_{\mathrm{o}}=h^{3} r^{-2} \\
\left(\mathrm{~A} \cdot \mathrm{m}^{-1}\right)\end{array}$ \\
\hline NiAAm673 & 13730 & 2.071 & 14740.1 & 24841 & 70551 \\
\hline NiAAm773 & 11154 & 2.282 & 14632.7 & 14316.6 & 13705 \\
\hline NiAAm873 & 1911 & 2.539 & 1977.23 & 2883.72 & 6134 \\
\hline
\end{tabular}

The values of $r$ and $h$, that were obtained by fitting the function given in Eq. 5 to the experimental data of heating rate $(\mathrm{d} T / \mathrm{d} t)_{t=0}$, are listed in Table 3 .

The value $n>2$ can be interpreted as a result of the size of magnetic particles in the sample which are considerably greater than those of typical superparamagnetic particles. The greater size of the magnetic particles leads to a release of thermal energy as a result of the additional loss caused by the hysteresis. In the case of the sample pyrolyzed at $673 \mathrm{~K}$, for which $n \cong 2$, a considerable part of the released thermal energy is a result of relaxation looses. However, the losses caused by the phenomenon of magnetic hysteresis dominate in the sample obtained at $873 \mathrm{~K}$.

From Eq. 5 it follows that the value of the magnetic field strength $H_{0}$, at which both components make an equal contribution to the power loss in the sample, in the magnetic field, amounts to

$$
H_{0}=\frac{h^{3}}{r^{2}} \text {. }
$$

For $H>H_{0}$, the losses related to magnetic hysteresis definitely start dominating in the total energy losses. The values of $H_{0}$, for the sample studied, are listed in Table 3.

From Eq. 5 the contribution of relaxation and hysteresis processes to the total release of thermal energy for the three samples were obtained 


$$
\frac{P_{\text {rel }}}{P_{\text {total }}}=\frac{h^{3}}{h^{3}+H r^{2}}, \quad \frac{P_{\text {hys }}}{P_{\text {total }}}=\frac{H r^{2}}{h^{3}+H r^{2}},
$$

which is graphically shown in Fig. 7.

Additional calorimetric measurements (Fig. 8) were also performed (at $H_{\mathrm{AC}}=$ $4000 \mathrm{~A} \cdot \mathrm{m}^{-1}$ ) versus frequency in the range from $20 \mathrm{kHz}$ to $800 \mathrm{kHz}$, which indicated that for the NiAAm673 sample, the heating process is the most effective for $f_{\mathrm{c}}=450$ $\mathrm{kHz}$. For the samples pyrolyzed at higher temperatures, the difference in $f_{\mathrm{c}}$ did not exceed $7 \%$; thus, the measurements were executed applying the same frequency, i.e., $450 \mathrm{kHz}$.

Taking into consideration that for immobilized magnetic grains, $\tau_{\mathrm{B}} \rightarrow \infty$, this means that $\tau_{\text {eff }}=\tau_{\mathrm{N}}=354 \mathrm{~ns}$. Knowing the value of the anisotropy constant for a magnetic material $K$ [18], it is possible to determine the size of magnetic crystallites.
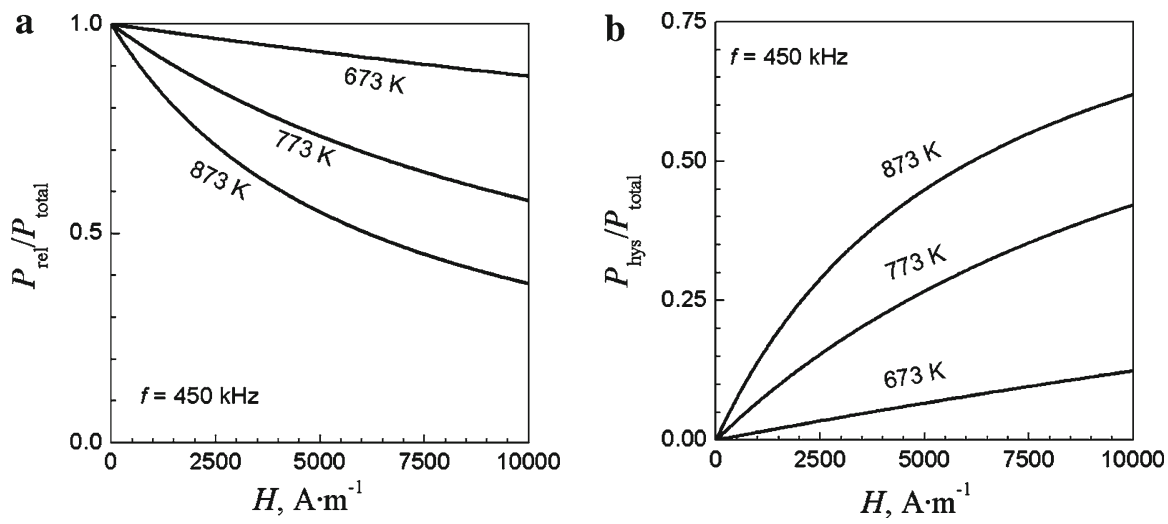

Fig. 7 Contribution of relaxation and hysteresis processes to the total release of thermal energy for the three samples as a function of the magnetic field strength: (a) relaxation and (b) hysteresis

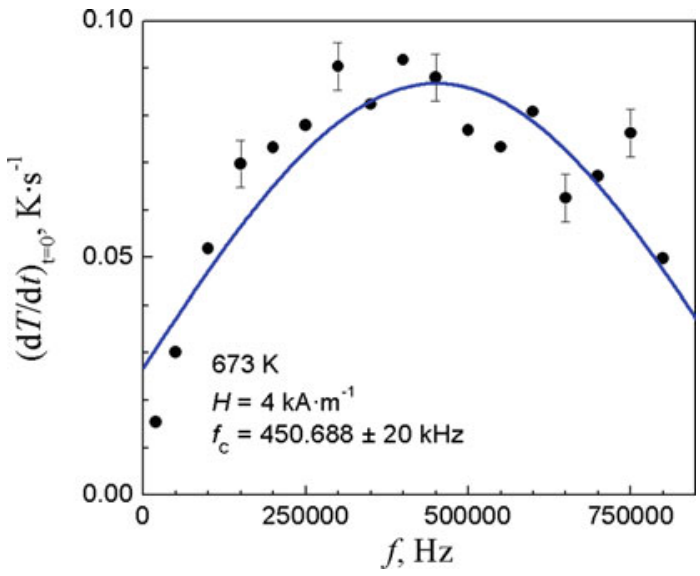

Fig. 8 Dependence of $(\mathrm{d} T / \mathrm{d} t)_{t=0}$ on the frequency at the alternating magnetic field strength $H_{\mathrm{AC}}=$ $4000 \mathrm{~A} \cdot \mathrm{m}^{-1}$, obtained for a sample pyrolyzed at $673 \mathrm{~K}$ (• experimental points, _ Gaussian function) 
In the second part of Eq. 1 , where $\tau_{\mathrm{o}}=1 \mathrm{~ns},|K|=5600 \mathrm{~J} \cdot \mathrm{m}^{-3}, k_{\mathrm{B}}=1.38 \times$ $10^{-23} \mathrm{~J} \cdot \mathrm{K}^{-1}$, and $T \cong 300 \mathrm{~K}$, we can obtain an average value of the crystallite diameter $\langle d\rangle$ :

$$
\langle d\rangle=\sqrt[3]{\frac{6 k_{\mathrm{B}} T \ln \left(\frac{\tau_{\mathrm{N}}}{\tau_{\mathrm{o}}}\right)}{\pi|K|}} \cong 20.2 \mathrm{~nm} .
$$

\section{Conclusions}

On the basis on the obtained results, several conclusions can be drawn:

- A three-step procedure consisting of frontal polymerization of a Ni acrylamide nitrate complex, followed by its pyrolysis, enables fabrication of magnetic beads containing metallic nanocrystallites embedded in a carbon matrix.

- The size of the crystallites, their morphology, volume fraction, and magnetic properties can be tailored by the pyrolysis temperature.

- The beads processed by pyrolysis at $673 \mathrm{~K}$ and $773 \mathrm{~K}$ exhibit a substantial contribution from superparamagnetic nanocrystallites, whereas the material pyrolyzed at the highest temperature, leads to a change of the magnetic ordering and the beads exhibit a behavior characteristic of a ferromagnetic material.

- The size of the crystallites affects their behavior in an external magnetic field, i.e., the heating process, which is the most effective for the sample pyrolyzed at $873 \mathrm{~K}$.

- The revealed $H^{\mathrm{n}}$-type dependence of the temperature increase rate, $(\mathrm{d} T / \mathrm{d} t)_{t=0}$, on the amplitude of the magnetic field indicates the presence of both superparamagnetic and ferromagnetic particles in all samples studied since $n>2$. For the superparamagnetic particles, the heating mechanism is associated with Néel relaxation.

- For the lower values of the magnetic field strength, $H<H_{0}$, the relaxation losses dominate, whereas for the opposite case, $H>H_{0}$, the magnetic hysteresis is the main source of thermal energy losses.

- Significant values of magnetic properties, such as coercivity and remanence, give a substantial contribution to energy losses for hysteresis, which for the sample pyrolyzed at $873 \mathrm{~K}$ was confirmed in a hyperthermal experiment.

- The composites containing magnetic Ni nanocrystallites entrapped in a carbon matrix can be potentially applied for hyperthermia treatment.

Acknowledgment Financial support from the Ministry of Science and Higher Education (Grant No. N N507 276136) is gratefully acknowledged.

Open Access This article is distributed under the terms of the Creative Commons Attribution Noncommercial License which permits any noncommercial use, distribution, and reproduction in any medium, provided the original author(s) and source are credited. 


\section{References}

1. J.P. Fortin, C. Wilhelm, J. Servais, C. Ménager, J.-C. Bacri, F. Gazeau, J. Am. Chem. Soc. 129, 2628 (2007)

2. R.E. Rosensweig, J. Magn. Magn. Mater. 252, 370 (2002)

3. R. Hergt, R. Hiergeist, M. Zeisberger, G. Glöckl, W. Weitschies, L.P. Ramirez, I. Hilger, W.A. Kaiser, J. Magn. Magn. Mater. 280, 358 (2004)

4. R. Hergt, W. Andrä, C.G. d'Ambly, I. Hilger, W.A. Kaiser, U. Richter, H.-G. Schmidt, IEEE Trans. Magn. 34, 3745 (1998)

5. R. Hiergeist, W. Andrä, N. Buske, R. Hergt, I. Hilger, U. Richter, W.A. Kaiser, J. Magn. Magn. Mater. 201, 420 (1999)

6. L. Rayleigh, Philos. Mag. 23, 255 (1887)

7. A.D. Pomogailo, G.I. Dzhardimaleva, Polym. Sci. Ser. A 3, 250 (2004)

8. A. Skumiel, J. Magn. Magn. Mater. 307, 85 (2006)

9. A.A. El-Gendy, E.M.M. Ibrahim, V.O. Khavrus, Y. Krupskaya, S. Hampel, A. Leonhardt, B. Büchner, R. Klingeler, Carbon 47, 2821 (2009)

10. F.E. Kaelble (ed.), Handbook of X-Rays (McGraw Hill, New York, 1967)

11. M. Kakudo, N. Kasai, X-Ray Diffraction of Polymers (Elsevier, Amsterdam, 1972)

12. M. Leonowicz, M. Wozniak, Y.M. Shulga, V.E. Muradyan, Z. Liu, H.A. Davies, W. Kaszuwara, J. Grabski, J. Magn. Magn. Mater. 294, 57 (2005)

13. T.A. Ngo, P. Bonville, P.M. Pileni, J. Appl. Phys. 89, 3370 (2001)

14. A. Ślawska-Waniewska, P. Didukh, J.M. Greneche, P.C. Fannin, J. Magn. Magn. Mater. 215(216), 227 (2000)

15. U. Voskoboynik, Acta. Phys. Pol. A 92, 43 (1997)

16. F. D’Orazio, F. Lucari, M. Melchiorri, C. Julián Fernández, G. Mattei, P. Mazzoldi, C. Sangregorio, D. Gatteschi, D. Fiorani, J. Magn. Magn. Mater. 262, 111 (2003)

17. A. Skumiel, Int. J. Thermophys. 31, 546 (2010)

18. P.A. Guimarães, Principles of Nanomagnetism (Springer-Verlag, Berlin, Heidelberg, 2009) 